\title{
The Cinderella discipline: morphometrics and their use in botanical classification
}

Article

Accepted Version

Christodoulou, M. D. ORCID: https://orcid.org/0000-00030968-2311, Clark, J. Y. and Culham, A. ORCID:

https://orcid.org/0000-0002-7440-0133 (2020) The Cinderella discipline: morphometrics and their use in botanical classification. Botanical Journal of the Linnean Society, 194 (4). pp. 385-396. ISSN 0024-4074 doi:

https://doi.org/10.1093/botlinnean/boaa055 Available at https://centaur.reading.ac.uk/91282/

It is advisable to refer to the publisher's version if you intend to cite from the work. See Guidance on citing.

To link to this article DOI: http://dx.doi.org/10.1093/botlinnean/boaa055

Publisher: Oxford University Press

All outputs in CentAUR are protected by Intellectual Property Rights law, including copyright law. Copyright and IPR is retained by the creators or other copyright holders. Terms and conditions for use of this material are defined in the End User Agreement. 


\section{CentAUR}

Central Archive at the University of Reading

Reading's research outputs online 


\section{REVIEW}

2 TITLE: The Cinderella Discipline: Morphometrics and their use in botanical classification 3

4 SHORT TITLE: Morphometrics and their use

5

6 AUTHORS: Christodoulou, M.D. ${ }^{1}{ }^{2 *}$, Clark, J.Y. ${ }^{3}$, and Culham, A. ${ }^{2}$

7

81 Department of Statistics, University of Oxford, Oxford, UK

9

102 University of Reading Herbarium, School of Biological Sciences, University of Reading,

11 Whiteknights, Reading, UK

12

133 Department of Computer Science, Faculty of Engineering and Physical Sciences,

14 University of Surrey, Guildford, UK

15

16 * For correspondence e-mail: maria.christodoulou@stats.ox.ac.uk

17 


\section{ABSTRACT}

20 Between the 1960s and the present day, the use of morphology in plant taxonomy

21 suffered a major decline, in part driven by the apparent superiority of DNA-based

22 approaches to data generation. However, in recent years computer image

23 recognition has re-kindled the interest in morphological techniques. Linear or geometric

24 morphometric approaches have been employed to distinguish and classify a wide

25 variety of organisms; each has strengths and weaknesses. Here we review these

26 approaches with a focus on plant classification and present a case for the

27 combination of morphometrics with statistical/machine learning. There is a large

28 collection of classification techniques available for biological analysis and selecting the

29 most appropriate one is not trivial. Performance should be evaluated using

30 standardised metrics such as accuracy, sensitivity, and specificity. The gathering and

31 storage of high-resolution images, combined with the processing power of desktop

32 computers, makes morphometric approaches practical as a time- and cost-efficient

33 way of non-destructive identification of plant samples.

Keywords: Plant taxonomy, geometric morphometrics, linear morphometrics, statistical learning, machine learning, identification, classification, neural networks. 
38 In his keynote address during the $50^{\text {th }}$ anniversary of botany MSc training at the

39 University of Reading, Prof Vernon Heywood described a steady decline in the state of

40 botany teaching in the UK with a resulting loss of skills in the next generation of scientists.

41 With few institutions in the country offering training for young botanists, more and more

42 researchers enter plant taxonomy through the field of molecular systematics, never

43 learning the classic skills of a traditional botanist. Although great progress has been

44 made in the development of molecular tools, increasing the insight gained from laboratory methods, what used to be the beating heart of botany - morphology - has

46 lost some of its appeal. In our view this is because morphological data coding cannot

47 readily be made into a clear data generation pipeline in the same way as much molecular data can. We believe this to be because morphology requires more indepth knowledge and understanding of the organism prior to data collection than is required for DNA sequencing and that morphological variation is open-ended rather than with a fixed range of states as in DNA data. Whilst morphological data have lost favour in the construction of plant classification systems they have gained popularity in the study of evolution from variation in gross morphology of the centropogonid clade (Lobelioideae: Campanulaceae) (Lagomarsino et al., 2017), speciation despite consistent floral morphology in Myrcia DC. (Vasconcelos et al., 2019) though to detailed morphometric analysis of traits related to environment in Vriesea Lindl.

57 bromeliads (Neves et al., 2020).

The power of some of the more modern developments in morphometrics and statistical learning however can provide botanists with an extra toolbox to help them describe and quantify the variation that surrounds them. In this review we aim to make a case for the value of morphometrics, especially in combination with more sophisticated statistical methods, in a botanist's analytical toolbox - not to replace molecular

64 techniques but to add to them. Morphology is often one of the most directly accessible and intuitive data sources for taxonomic research. In botanical taxonomy, 
66 morphological characterization is the foundation of taxon description and

67 identification, albeit often found in the formal and stylised format present in Floras and

68 monographs. There is an opportunity for modern botanical taxonomy to explore the

69 rapidly advancing field of morphometrics which already has some notable examples

70 ranging from automatic leaf outline identification of Passiflora L. species (De Oliveira

71 Plotze \& Martinez Bruno, 2009), the tooth margin algorithm for Tilia L. leaf identification

72 (Corney et al., 2012), and the use of leaf venation architecture for major angiosperm

73 clade recognition (Wilf et al., 2016). Some computerised systems, starting with an

74 existing classification of taxa, can use machine learning to handle the routine

75 identification work, and then refer intransigent problems to a human expert (Clark,

76 Corney, \& Wilkin, 2017).

78 One of the principal arguments presented against morphological data is the potential 79 for high levels of ambiguity. This ambiguity can be caused by a variety of factors such 80 as inaccurate character definition (Assis, 2009) and difficulty in establishing homology

81 (Schneider, Smith, \& Pryer, 2009). Morphological data collection can be further

82 complicated by plasticity of features (Perkins, Martinsen, \& Falk, 2011), homoplasy

83 (Schneider et al., 2009), low numbers of characters (Giribet, 2010), and missing

84 character states (Jenner, 2004). In some organisms such as parasites, reduced body

85 plans can make characterisation of features even more difficult and lead to a very

86 limited dataset (Perkins et al., 2011). These concerns are neither exaggerated nor trivial

87 and many are thoroughly discussed in the morphological literature. They do not,

88 however, necessarily imply a lower quality of data produced by morphological work in

89 comparison with other data sources (Jenner, 2004).

91 As most botanical researchers are question-driven rather than method-driven, we have

92 structured our recommendations using general outlines on what kind of questions each

93 combination of morphological and statistical tools can answer, with the aim of 
94 promoting more thorough morphological investigation in botanical research. We have

95 split this into two sections - Developmental hypotheses and Classification hypotheses.

96 Under Developmental hypotheses we include all studies that may require the

97 description of shape or size of a plant either to compare between treatments or to

98 study how characters change along a particular gradient. For these we give an outline

99 of morphometric tools available. Under Classification hypotheses we include all studies

100 where the researcher is asking questions of taxon membership (e.g. are these two

101 groups in the same taxon?) or questions of identification (e.g. what is the minimum set

102 of diagnostics to accurately identify a sample?). These also require morphometric tools,

103 such as those described under the developmental hypotheses, but can be taken

104 further by combining them with machine learning techniques. There is a difference in

105 terminology between the use of the word classification in biology and in computer

106 science. Although the term is clearly defined in a taxonomic setting as the formal

107 structure in which taxa are placed, in machine learning it means something much

108 more general: it is the attribution of objects to a particular group. This is why

109 identification in the machine learning context falls under classification, and therefore is

110 included here under Classification hypotheses. 


\section{DEVELOPMENTAL HYPOTHESES}

112 Plant growth and development studies already rely heavily on morphological

113 measurements - size for example is often included as a proxy to an organism's

114 developmental stage. These studies often focus on examining how the organism

115 changes as it progresses through the various life stages. These could range from

116 progression from seed to flower for an annual, or even development of fruit on a tree

117 during the growing season.

119 Even though it is very commonly used, size itself is a complex and often unappreciated

120 concept. Often researchers fail to explore the separation between shape and size,

121 confounding the two and losing some of the clarity that can be obtained through their 122 investigation. For Developmental hypotheses, we argue that the crucial point for insight 123 is not the separation of size and shape just for the sake of it - it is for the researcher to 124 either knowingly combine them or distinguish between the two based on the 125 hypothesis in question. We believe that by cautiously selecting measurements that do 126 not distinguish shape from size, a researcher can gain insight on changes in either size 127 or shape during a developmental process based on how they use them. For example, 128 the length of apple fruit through the growing season, plotted against time from anthesis 129 can give insight on how size develops as time progresses (Atay, Pirlak, \& Atay, 2010). 130 The ratio between length and width for the same fruit provides an indication of the 131 development of shape (Bollard, 1970). Both length and width independently are size 132 metrics, but in combination they describe shape.

134 In the context of describing morphology, there are two mainstream methods: linear 135 and geometric morphometrics. An essential distinction between them is that linear 136 morphometrics do not actively separate size from shape, whereas geometric 137 morphometrics do. We have structured the remainder of this section to describe these 138 two techniques and have illustrated them using biological examples. 
140 The traditional approach to morphometrics involves the measurement of distances

141 between points deemed to be characteristic of shape and form. Measurements such

142 as height, length, width, and diameter all fall under the general categorisation of linear

143 morphometrics. These measurements are intuitive, easy to understand and to interpret,

144 and have been in the biological toolbox for as long as the toolbox itself has existed.

145 Linear morphometrics are quick to collect, low cost, easy to interpret, and often

146 sufficient for biological description. Sanchez et al. (2011) compared the growth

147 development of baobab seedlings of different origins using a variety of morphometric

148 measurements, such as length and diameter of roots, to establish that plants originating

149 from drier environments grew to a smaller size even under optimal greenhouse

150 conditions. Richardson et al. (2011) the studied fruit development patterns of kiwi from

151 anthesis to ripening using amongst other character a collection of linear

152 morphometrics, such as pericarp diameter. Zhang et al. (2015) performed a

153 comparative study of the developmental patterns of Sweet cherry floral parts, using

154 linear measurements such as pedicel length, establishing a correlation between floral

155 morphology and environmental conditions during growth such as temperature.

157 For morphometric studies that require the description of very subtle shape characters,

158 linear morphometrics may not be the most appropriate tool. The reason for this is

159 because distance measurements, although excellent for summarising shape and size

160 descriptions, often lack context. To correct for this, more linear measurements can be

161 collected, creating a more complete dataset for each object. When the shape of

162 interest is of biological form, it becomes crucial to be able to establish and quantify

163 even the subtlest of differences. To be able to achieve this through linear

164 morphometrics would involve an extensive collection of measurements and a

165 generous amount of luck, as one may simply fail to measure the precise point where

166 differences between taxa occur. Furthermore, within an evolutionary framework it is 
more appropriate to view form as a whole since organisms evolve as a whole. To

168 counter these concerns, morphometric theory progressed to what is often described as

169 modern morphometrics, more accurately known as geometric morphometrics.

171 Geometric morphometrics allow the study of the shape of an organism as a whole,

172 rather than as a collection of separate components. In contrast to linear

173 morphometrics, by studying all the selected landmarks of a sample together, even

174 subtle changes in geometry can be quantified and analysed using geometric

175 morphometrics. Kendall's shape definition forms the basis of geometric morphometrics

176 (Zelditch et al., 2004). This clear separation of shape from position, orientation and size

177 corresponds to an intuitive concept of shape. In practical terms, to achieve this

178 separation there is a strong analytical reliance on multivariate techniques (Klingenberg

179 \& Monteiro, 2005). The way this is performed in geometric morphometrics is through the

180 use of landmark coordinates (Van Bocxlaer \& Schultheiß, 2010). A landmark is a

181 recognizable point on the organism that, together with other landmarks, can be used

182 to summarise the form of the organism (Zelditch et al., 2004). As opposed to focusing

183 on distance measurements, as is done in linear morphometrics, shape is summarised

184 through the Cartesian coordinates of selected landmarks (Walker, 2000). By always

185 analysing these coordinates together in a multidimensional space, shapes can be

186 scaled, moved and rotated without losing any information (Goodall, 1991). Although

187 the selection of appropriate landmarks can be difficult, this multivariate approach

188 provides great flexibility for manipulation and statistical analysis.

190 After landmark selection, the recording of coordinates for all samples (a process

191 referred to as "sample digitisation") creates the initial dataset to be used for analysis.

192 The samples in this dataset are not, however, comparable if their coordinates have not

193 been standardised. This is because regardless of how carefully and methodically

194 digitisation occurred the samples are bound to not be fully aligned. Furthermore, 
differences in sizes between samples will affect the position of the landmarks on the

196 Cartesian axes, confounding shape comparison. To correct for this, the samples can be

197 standardised using a Procrustes superimposition (Rohlf \& Slice, 1990). Named after the

198 mythical ancient Greek bandit who trimmed or stretched his victims to fit an iron bed,

199 the process superimposes the samples using the landmarks to correct for orientation

200 and alignment (Stegmann \& Gomez, 2002). It then proceeds to stretch or shrink some

201 samples aiming for all samples to be perfectly superimposed (Zelditch et al., 2004). We

202 have illustrated the steps of this process in Figure 1.

Selecting appropriate landmarks to summarise a shape is perhaps the most crucial

aspect of geometric morphometrics. The reason for this is that if the choice of landmarks is poor, then any subsequent analysis will reflect that. Through the process of landmark selection, the overall shape of the organism in question is summarised using a small number of representative landmarks. Selecting representative landmarks is a subjective exercise that relies on in-depth knowledge and understanding of anatomy and biology of the organism in question. This is because not all landmarks are created equal. A wisely chosen landmark can summarise shape appropriately and provide adequate information for biological inference. A poorly selected landmark will at best add high levels of noise to the dataset or, at worst, result in misleading patterns.

215 Ideally, landmark selection requires four criteria that ensure quality: repeatability,

216 consistency of position, adequacy and homology (Zelditch et al., 2004). Repeatability

217 refers to the potential of locating the selected landmark accurately on a specimen

218 multiple times (Zelditch et al., 2004). If a landmark is difficult to locate or its position is

219 relatively vague, then samples that have no significant biological differences may be

220 found to be different as an artefact of poor landmark choice. Consistency of position

221 refers to the relative positions between landmarks (Zelditch et al., 2004). If two

222 landmarks switch relative positions between different specimens then their comparison 
can lead to statistical outliers that may affect the findings and analyses. Adequacy

224 refers to the number and position of landmarks used to summarise a form (Zelditch et

225 al., 2004). Although more is not always better in terms of landmark selection, including

226 too few landmarks will not lead to a representative dataset. Even though repeatability

227 can be quantified, and consistency of position detected, adequacy is a harder

228 criterion to evaluate. This is because adequate coverage can be highly subjective. The

229 concept relies on finding the golden mean between oversampling the specimen

230 (where too many landmarks can lead to higher noise levels in the dataset) and

231 undersampling (losing possible detectable variation between specimens).

233 Homology in landmark selection has both geometric and biological aspects. Two landmarks are considered homologous in two specimens if there is a degree of correspondence between them. This correspondence can be purely a geometric attribute (e.g. the tips of the Giza pyramids are geometrically homologous) or a biological attribute (e.g. the forelimbs of bats and primates). Although all four criteria are important for landmark quality, establishing homology is crucial. It is only through

239 the use of homologous landmarks that the shapes studied are truly comparable. If the 240 landmarks used are not homologous between the organisms in the study then there is no logical support for their comparison and the results can be highly misleading

242 (Klingenberg, 2008). Although homology is considered one of the most crucial aspects

243 in landmark selection, exactly how it can affect a given study depends on the nature

244 and scope of the study itself. In general, the ability to identify homology can severely

245 limit the quantity of potential landmark candidates. These constraints imposed by homology increase the popularity of outline methods of analysis (Macleod, 1999). By replacing homologous landmarks with regularly spaced points along a curve, outline analysis sidesteps the issue of homology and can be used

250 in cases where landmarks are sparse or hard to define (Macleod, 1999). Outline data 
can then be analysed using Fourier harmonics (or possible variations such as Elliptical

252 Fourier) or Eigenshape analysis (Macleod, 1999; Bonhomme \& Claude, 2014). Although

253 outline analysis is a popular and successful alternative to landmark analysis, the

254 assumption that it bypasses homology issues may be misplaced. The reason for this is

255 that outline methods are not completely independent of landmark correspondence

256 assumptions (Klingenberg, 2008). That is because as with landmark methods, outline

257 coordinates require a superimposition technique, such as Procrustes superimposition,

258 prior to analysis (Bonhomme \& Claude, 2014). This means that the outline points that

259 are recorded are treated as actual homologous landmarks. This may appear minor,

260 but as the superimposition process assumes a certain correspondence between points

261 on the outline, it can result in increased levels of noise in the dataset. Furthermore,

262 analytical approaches such as Elliptic Fourier Analysis also assume a certain degree of

263 homology between outline points. It can therefore be argued that the principal

264 difference between the two approaches is that in landmark analysis the homology

265 criterion is explicit whereas in outline analysis it is implied and often ignored.

267 The choice between linear and geometric morphometrics for an analysis is not trivial as

268 one technique is not necessarily superior to the other. Linear morphometrics are quick,

269 intuitive and cost effective and often robust enough to not introduce noise in the

270 analysis. They fail when separation of shape and size becomes important and when

271 subtle changes in morphology are crucial - this is where geometric morphometrics

272 excel. Selecting the appropriate method for the question in hand is always a

273 challenging aspect of scientific discovery, although familiarity with both methods,

274 combined with understanding of the studied organism helps when deciding which

275 technique may provide more insightful findings. As a final point, it is not always

276 necessary to choose one over the other, for example, Christodoulou et al. (2018)

277 combined linear and geometric morphometrics to describe shape differences

278 between apple cultivars with greater accuracy. 
280 Although classification in biology has a different meaning than in machine learning (a 281 subset of statistical learning), this collection of hypotheses relies on grouping objects based on similarities between measured characters. These can include studies of morphological similarities between geographically distinct populations, segregation between species and hybrids, or revision of taxonomic limits.

Both linear and geometric morphometrics have been used for such studies. Compton and Hedderson (1997), in their taxonomic revision of the limits of Cimicifuga foetida L. s.l. (now Actaea cimicifuga L.), included 17 length variables, resulting in the detection of four geographically distinct species. Blanco-Dios (2007) used multivariate analysis of 17 linear morphometric characters to contrast the morphology of hybrid populations between Armeria beirana Franco and A. pubigera (Desf.) Boiss. with that of their progenitors, detecting clear differences between the groupings. Da Costa et al. (2009) used distance measurements for both vegetative and reproductive parts to study the variation within the Vriesea paraibica Wawra complex. After statistical analysis, they proceeded to recognise four species within the complex (V. paraibica, V. interrogatoria L.B.Smith, V. eltoniana E.Pereira \& Ivo, and V. flava A.F. Costa, H. Luther \& M.G.L. Wanderley), for which they provided a taxonomic treatment. Returning to the genus Actaea L., Gardner et al. (2012) used linear morphometrics to quantify the variation within Actaea racemosa L., establishing that between-population variation was similar to within-population variation. In a study of the Andropogon lateralis Nees complex, Nagahama et al. (2014) used 19 linear morphometric measurements to successfully distinguish both species and hybrids within the complex. Shipunov and Bateman (2005) used geometric morphometrics to explore the diversity of lip shapes of Dactylorhiza Neck. ex Nevski orchids, studying both hybridization patterns and taxonomy in Russian populations. Volkova and Shipunov (2007) used similar tools to investigate the variation between three Nymphaea L. species in Russia and Siberia, 
finding the species delimitation to be robust. Viscosi et al. (2009) successfully used

308 geometric morphometrics on oak leaves to distinguish between four species. Savriama

309 et al. (2012) presented a new methodology quantifying symmetry and asymmetry of

310 corolla shape in Erysimum mediohispanicum Polatschek (now Erysimum grandiflorum

311 subsp. mediohispanicum (Polatschek) Romo), establishing symmetry to be a

312 fundamental character for floral variation within the taxon. Finally, Fernández-

313 Mazuecos et al. (2013) used geometric morphometrics to study the role of flower

314 specialisation for speciation in Linaria Mill. subsect. Versicolores (Benth.) Wetst. finding

315 corolla tube differences to correlate with divergent pollination strategies. In a

316 comparison of leaf shape of Anacardium microcarpum Ducke with A. occidentale L.

317 using geometric morphometric descriptors, Vieira et al. (2014) established that

318 although the leaves do present statistically significant differences, overlap between

319 taxa and populations prevent them from being used as unique identifiers.

Analytically, methods from statistical/machine learning can offer great insight for this type of hypothesis. There are two broad sections in statistical/machine learning: supervised learning and unsupervised learning. We are excluding deep learning methods here, as the topic is too large for an adequate description within this review and the approaches are rather different. The review on the topic by Angermueller et al. (2016) offers a good overview of the major issues. Furthermore, deep learning is primarily aimed at processing huge amounts of multivariate data (so called 'big data'), and here we are more concerned with the utilisation of relatively small datasets, often with only a few data records per taxon, which is more realistic for consideration by practising botanists.

Supervised learning focuses on using combinations of characters to circumscribe known groups (classes) and then applying this knowledge to predict the class membership of an unknown sample (Tarca et al., 2007). This is essentially 'identification' 
in the biological sense, if the classes represent named taxa. The classic example of

336 supervised learning is Anderson's Iris dataset analysed by Fisher using Linear

337 Discriminant Analysis (LDA) (Fisher, 1936). The original dataset contained measurements

338 from 150 flowers belonging to three Iris species (50 flowers each of I. setosa Pall. ex Link,

339 I. versicolor Thunb. and I. virginica L.), For each flower, length and width measurements

340 of two tepals (one inner, and one outer tepal), as well as species, were recorded.

341 When this dataset was analysed using LDA, discriminant functions were established for

342 each species based on the lengths and widths of the tepals. These could then be used

343 to establish the species of an unknown Iris sample using only length and width tepal

344 measurements (provided it belonged to one of the three species). The factor that

345 makes this example part of supervised learning is the prior knowledge of class

346 membership, in this case Iris species, used for the design of the discriminant functions

347 (Fogel, 2008).

349 Unsupervised learning, by contrast, has no prior knowledge of class membership, and

350 the analysis aims to explore patterns in the data and create natural groupings (Fogel,

351 2008). Such groupings can then be used as justification for delimitation of traditional

352 ranked taxa such as species. This is essentially 'classification' in the biological sense.

353 Cluster analysis (clustering), for example, is a case of unsupervised learning. Table 1

354 summarises a selection of both supervised and unsupervised techniques, more

355 extensive descriptions of which can be found in Appendix A.

357 Table 1 showcases botanical applications of machine learning. The combination of

358 machine learning and morphometrics for classification has much more prominent

359 examples outside of botany. We aim for this review to increase the uptake of these

360 techniques in botany. In the meantime, we present some non-botanical examples here

361 for illustration purposes. Santana et al. (2014) studied bee classification using the

362 forewings of male members of five Euglossa species. This was performed by using 18 
landmarks on the wing venation together with colour change variables, followed by

364 comparisons between classification techniques including linear discriminant analysis

365 and a modified neural network. The neural network outperformed the other classifiers,

366 with an accuracy of $87.6 \%$. da Silva et al. (2015) used more classes than Santana et al.

367 (2014), studying 26 subspecies of Apis mellifera while still using the same 18 landmarks

368 on wing venation. Their focus was on the performance of feature selection and their

369 conclusion was that a Naïve Bayes classifier outperforms other classification techniques,

370 with $65 \%$ mean accuracy on cross-validation (da Silva et al., 2015).

372 Van Bocxlaer and Schultheiß's (2010) gastropod study was one of the first in zoology to

373 combine machine learning with morphometrics, their focus was primarily on comparing

374 landmark analysis with outline analysis. For their gastropod dataset they found that

375 outline analysis outperformed landmark analysis by $3 \%$, reaching $78 \%$ accuracy when

376 using a Support Vector Machine (SVM) classification (Van Bocxlaer \& Schultheiß, 2010).

377 The high success rate of the outline analysis is likely due to the presence of three-

378 dimensional ornamentation on the shell surface. Also, the theory of outline methods for

379 biological shape analysis is not as robust as landmark analysis, as discussed briefly in

380 earlier sections.

382 Guisande et al. (2010) describe new software designed to identify fish species, using

383 Classification and Regression Trees (CARTs) and linear morphometrics. The structure of

384 the software is such that the user is required to make linear measurements on their

385 sample, following a certain protocol, and the measurements are then used to classify

386 the sample. This makes it similar to a multi-access key rather than a tool for automatic

387 identification. For multi-access keys, success rates can be established by testing the key

388 on the target audience and recording how successful was their navigation of the key.

389 Guisande et al. (2010) did not perform this test and only tested accuracy using samples

390 they had measured themselves. 
392 In the field of anthropology, Velemínská et al. (2013) used semi-landmarks to study the 393 greater sciatic notch (which is part of the pelvis bones) aiming to correctly classify the 394 sex of the individual. Their best performing classifier was a Support Vector Machine that achieved a $92 \%$ accuracy. Instead of using a completely independent test set, the accuracy was quantified using a leave-one-out cross-validation approach on the learning set. The absence of a separate test set can lead to overestimating the accuracy of the classification as briefly discussed earlier.

The orthodontics paper by Yu et al. (2014) is based on the unusual premise of predicting attractiveness on malocclusion patients (patients with misaligned teeth). By using 101 landmarks on patient images combined with a Support Vector Machine, they

403 achieved an accuracy of attractiveness prediction of $72 \%$. This work is interesting 404 because it is the only example in the literature where geometric morphometrics have been combined with the regression approaches of statistical learning, rather than the classification ones. This is because the attractiveness measure used was based on a

407 (subjective) score from 69 orthodontics experts, therefore the prediction was a

408 continuous measurement rather than a class.

409

\section{Model evaluation}

411 There is a large collection of classification techniques available for biological analysis

412 and selecting the most appropriate technique is not trivial. The reason for this is that

413 there is no single classification technique that consistently outperforms all others

414 regardless of the dataset studied. In machine learning this concept is referred to as the

415 "No free lunch" Theorem. Stated formally by Wolpert and Macready (1997), the

416 theorem suggests that the performance of all classifiers is equal when the totality of

417 possible problems is considered. This means that for every classifier available there exists

418 a possible problem where that classifier outperforms every other classifier. In practical 
419 terms, this makes selecting a classifier for a study harder as the only way to establish the 420 appropriateness of the technique is after the training of the classifier. Due to this, the 421 most common approach to classification problems is to train a variety of different 422 classifiers and then select the one that performs best (Fogel, 2008). This strategy makes performance evaluation the focus of the classification analysis. To this extent a series of

424 metrics have been proposed in the literature, summarised in Table 2.

426 All the metrics presented in Table 2 rely on describing classification success through the 427 use of a set of samples, however selecting the set that is used is not straightforward. In 428 most biological situations there is a limited amount of data available for study, making 429 each individual sample valuable to the study. With a limited dataset, therefore, the 430 decision on the appropriate "spending" of the data is not an easy one to make. This 431 makes pilot studies that can inform power analyses (to estimate appropriate sample sizes) a crucial aspect of experimental design (McDonald, 2014).

434 There are three stages in machine learning that require data: training, validating and 435 testing (Olden, Lawler, \& Poff, 2008). During the first stage the classifier is primarily 436 trained to the problem in question. If the whole dataset is used at this stage then it will 437 have to be re-used for both validating and testing, leading to potential overfitting and 438 unrealistically high performance metrics (Olden et al., 2008). This is because the 439 classifier would have knowledge of the full dataset at the training stage, therefore 440 when validating occurs (which is the process that verifies that appropriate tuning 441 parameters have been selected during training), overfitting is more likely as none of the 442 validating samples will be new. When the classifier is then tested using known samples, 443 the performance will appear improved due to this overfitting effect. The peril from this is 444 that when the classifier is applied to truly unknown samples, the confidence in the 445 resulting class could be misplaced. To avoid this, common practice involves partitioning 446 the initial dataset to a training set (including a validation set) and a testing set. In this 
case the testing set is used solely for establishing the final, unbiased, performance of

448 the classifier (Olden et al., 2008). As this partition reduces the data available for training

449 and validating, partitioning the training dataset further may not be realistic as an inappropriately small training set will create an inappropriate and untrustworthy

451 classifier.

In order to reduce overfitting during the validating process, cross-validation (CV) can be used instead. In cross-validation the training dataset is partitioned, creating a

455 training set (in the strict sense) and a validation set (Olden et al., 2008). Training

456 commences and is terminated when the performance with respect to the validation set begins to reduce. The validation set is thus used as a dummy 'test' set. After the classifier is trained and validated the two datasets are re-combined and re-partitioned

459 creating a new training and validation dataset. The learning process is repeated again 460 from the start until either a predefined number of data partitions, or all possible data partitions, have been used for training. In biological applications of machine learning, multifold (K-fold) cross-validation is commonly used to help avoid overfitting (Olden et al., 2008). During that process the training dataset is partitioned into $\mathrm{K}$ equal sets, with $\mathrm{K}$ 1 of these recombined to create the training set and the last one used to validate. This process is repeated $\mathrm{K}$ times for all possible (or sensible) combinations of training and validation sets. More recently this technique has been slightly modified to include

467 further repetitions; for example, in $\mathrm{M}$ repetitions of $\mathrm{K}$-fold cross-validation the process of K-fold cross-validation already described is repeated $M$ times. An example using two repetitions of 5 -fold cross-validation is illustrated in Figure 2.

471 Throughout this paper, we have explained and illustrated the many strengths of 472 morphometric study including the ability to train and evaluate a system, to conduct 473 power analysis on trial data sets to help decide on appropriate sample sizes and the 474 crucial element of reproducible measurement. Morphometric approaches can offer to 
475 build strong and reproducible systems of classification and these can be combined

476 with DNA derived data to give a holistic synthesis that might improve the stability and

477 decrease the subjectivity of plant classification, especially at the species level. In short,

478 when botanists and horticulturalists catch up with other disciplines we expect to see

479 use of morphological data in the construction of more robust botanical classification

480 systems.

481 


\section{REFERENCES}

483 Angermueller C, Pärnamaa T, Parts L, Stegle O. 2016. Deep learning for computational 484 biology. Molecular Systems Biology 12: 878.

485 Assis LC. 2009. Coherence, correspondence, and the renaissance of morphology in 486 phylogenetic systematics. Cladistics 25: 528-544.

487 Atay E, Pirlak L, Atay A. 2010. Determination of fruit growth in some apple varieties. Journal of Argicultural Sciences 16: 1-8.

Blanco-Dios JB. 2007. Estudio morfométrico de una zona híbrida entre Armeria beirana y A . pubigera ( Plumbaginaceae ) en el noroeste de la Península lbérica. Anales del Jardín Botánico de Madrid 64: 229-235.

Van Bocxlaer B, Schultheiß R. 2010. Comparison of morphometric techniques for shapes with few homologous landmarks based on machine-learning approaches to biological discrimination. Paleobiology 36: 497-515.

Bollard EG. 1970. The physiology and nutrition of developing fruit. In: Hulme AC, Rhodes MJ, eds. The biochemistry of fruit and their producs: Volume I., 387-425.

Bonhomme V, Claude J. 2014. Momocs: Outline analysis using R. Journal of Statistical Software 56: 1-24.

Christodoulou, M. D., Battey NH, Culham A. 2018. Can you make morphometrics work when you know the right answer? Pick and mix approaches for apple identification.

Clark JY, Corney D, Wilkin P. 2017. Leaf-based automated species classification using image processing and neural networks. In Lestrel P, ed. Biological Shape Analysis Proceedings of the $4^{\text {th }}$ International Symposium: 29-56. World Scientific, Singapore.

Compton JA, Hedderson TA. 1997. A morphometric analysis of the Cimicifuga foetida L. complex (Ranunculaceae). Botanical Journal of the Linnean Society 123: 1-23.

Corney DPA, Tang HL, Clark JY, Hu Y, Jin J. 2012. Automating digital leaf measurement:

507 The tooth, the whole tooth, and nothing but the tooth. PLOS ONE 7: 1-10.

508 Da Costa AF, Rodrigues PJFP, Wanderley MDGL. 2009. Morphometric analysis and 509 taxonomic revision of the Vriesea paraibica complex (Bromeliaceae). Botanical 
Journal of the Linnean Society 159: 163-181.

511 Cuni Sanchez A, De Smedt S, Haq N, Samson R. 2011. Variation in baobab seedling

512 morphology and its implications for selecting superior planting material. Scientia

513 Horticulturae 130: 109-117.

514 Fernández-Mazuecos M, Blanco-Pastor JL, Gómez JM, Vargas P. 2013. Corolla

515 morphology influences diversification rates in bifid toadflaxes (Linaria sect.

516 Versicolores). Annals of Botany 112: 1705-1722.

517 Fisher R. 1936. The use of multiple measurements in taxonomic problems. Annals of

518 Eugenics 7: 179-188.

519 Fogel GB. 2008. Computational intelligence approaches for pattern discovery in

520 biological systems. Briefings in Bioinformatics 9: 307-316.

521 Gardner ZE, Lueck L, Erhardt EB, Craker LE. 2012. A morphometric analysis of Actaea

522 racemosa L. (Ranunculaceae). Journal of Medicinally Active Plants 1: 47-59.

523 Giribet G. 2010. A new dimension in combining data? The use of morphology and

524 phylogenomic data in metazoan systematics. Acta Zoologica 91: 11-19.

525 Goodall C. 1991. Procrustes Methods in the Statistical Analysis of Shape. Journal of the

526 Royal Statistical Society. Series B (Methodological) 53: 285-339.

527 Guisande C, Manjarrés-Hernández A, Pelayo-Villamil P, Granado-Lorencio C, Riveiro I,

528 Acuña A, Prieto-Piraquive E, Janeiro E, Matías JM, Patti C, Patti B, Mazzola S, Jiménez S,

529 Duque V, Salmerón F. 2010. IPez: An expert system for the taxonomic identification of

530 fishes based on machine learning techniques. Fisheries Research 102: 240-247.

531 Jenner RA. 2004. When molecules and morphology clash: Reconciling conflicting

532 phylogenies of the Metazoa by considering secondary character loss. Evolution and

533 Development 6: 372-378.

534 Klingenberg CP. 2008. Novelty and 'homology-free' morphometrics: What's in a name?

535 Evolutionary Biology 35: 186-190.

536 Klingenberg CP, Monteiro LR. 2005. Distances and directions in multidimensional shape

537 spaces: implications for morphometric applications. Systematic Biology 54: 678-688. 
Lagomarsino LP, Forrestel EJ, Muchhala N, Davis CC. 2017. Repeated evolution of

539 vertebrate pollination syndromes in a recently diverged Andean plant clade. Evolution

$540 \quad 71: 1970-1985$.

541 Macleod N. 1999. Generalizing and extending the eigenshape method of shape space

542 visualization and analysis. Paleobiology 25: 107-138.

543 McDonald JH. 2014. Handbook of Biological Statistics. Baltimore, Maryland.: Sparky

544 House Publishing.

545 Nagahama N, Anton AM, Norrmann G a. 2014. Taxon Delimitation in the Andropogon

546 lateralis Complex (Poaceae) in Southern South America based on Morphometrical

547 Analyses. Systematic Botany 39: 804-813.

548 Neves B, Zanella CM, Kessous IM, Uribbe FP, Salgueiro F, Bered F, Antonelli A, Bacon CD,

549 Costa AF. 2020. Drivers of bromeliad leaf and floral bract variation across a latitudinal

550 gradient in the Atlantic Forest. Journal of Biogeography 47: 261-274.

551 Olden JD, Lawler JJ, Poff NL. 2008. Machine learning methods without tears: a primer for 552 ecologists. The Quarterly Review of Biology 83: 171-193.

553 De Oliveira Plotze R, Martinez Bruno O. 2009. Automatic Leaf Structure Biometry:

554 Computer Vision Techniques and their Applications in Plant Taxonomy. International

555 Journal of Pattern Recognition and Artificial Intelligence 23: 247-262.

556 Perkins SL, Martinsen ES, Falk BG. 2011. Do molecules matter more than morphology?

557 Promises and pitfalls in parasites. Parasitology 138: 1664-1674.

558 Richardson AC, Boldingh HL, McAtee PA, Gunaseelan K, Luo Z, Atkinson RG, David KM,

559 Burdon JN, Schaffer RJ. 2011. Fruit development of the diploid kiwifruit, Actinidia

560 chinensis 'Hort16A'. BMC Plant Biology 11.

561 Rohlf F, Slice D. 1990. Extensions of the Procrustes method for the optimal

562 superimposition of landmarks. Systematic Biology 39: 40-59.

563 Santana FS, Costa AHR, Truzzi FS, Silva FL, Santos SL, Francoy TM, Saraiva AM. 2014. A

564 reference process for automating bee species identification based on wing images

565 and digital image processing. Ecological Informatics 24: 248-260. 
Savriama Y, Gómez JM, Perfectti F, Klingenberg CP. 2012. Geometric morphometrics of

567 corolla shape: Dissecting components of symmetric and asymmetric variation in

568 Erysimum mediohispanicum (Brassicaceae). New Phytologist 196: 945-954.

569 Schneider H, Smith AR, Pryer KM. 2009. Is morphology really at odds with molecules in

570 estimating fern phylogeny? Systematic Botany 34: 455-475.

571 Shipunov AB, Bateman RM. 2005. Geometric morphometrics as a tool for understanding

572 Dactylorhiza (Orchidaceae) diversity in European Russia. Biological Journal of the

573 Linnean Society $85: 1-12$.

574 da Silva FL, Sella MLG, Francoy TM, Costa AHR. 2015. Evaluating classification and

575 feature selection techniques for honeybee subspecies identification using wing images.

576 Computers and Electronics in Agriculture 114: 68-77.

577 Stegmann M, Gomez DD. 2002. A brief introduction to statistical shape analysis.

578 Tarca AL, Carey VJ, Chen X wen, Romero R, Drăghici S. 2007. Machine learning and its

579 applications to biology. PLOS Computational Biology 3: 0953-0963.

580 Vasconcelos TNC, Chartier M, Prenner G, Martins AC, Schönenberger J, Wingler A,

581 Lucas E. 2019. Floral uniformity through evolutionary time in a species-rich tree lineage.

582 New Phytologist 221: 1597-1608.

583 Velemínská J, Krajíček V, Dupej J, Goméz-Valdés JA, Velemínský P, Šefč́áková A,

584 Pelikán J, Sánchez-Mejorada G, Brưžek J. 2013. Technical Note: Geometric

585 morphometrics and sexual dimorphism of the greater sciatic notch in adults from two

586 skeletal collections: The accuracy and reliability of sex classification. American Journal

587 of Physical Anthropology 152: 558-565.

588 Vieira M, Mayo SJ, de Andrade IM. 2014. Geometric morphometrics of leaves of

589 Anacardium microcarpum Ducke and A. occidentale L. (Anacardiaceae) from the

590 coastal region of Piauí, Brazil. Revista Brasileira de Botanica 37: 315-327.

591 Viscosi V, Fortini P, Slice DE, Loy A, Blasi C. 2009. Geometric morphometric analyses of

592 leaf variation in four oak species of the subgenus Quercus (Fagaceae). Plant

593 Biosystems - An International Journal Dealing with all Aspects of Plant Biology 143: 575- 
595 Volkova PA, Shipunov AB. 2007. Morphological variation of Nymphaea

596 (Nymphaeaceae) in European Russia. Nordic Journal of Botany 25: 329-338.

597 Walker J. 2000. Ability of geometric morphometric methods to estimate a known

598 covariance matrix. Systematic Biology 49: 686-696.

599 Wilf $\mathbf{P}$, Zhang S, Chikkerur S, Little SA, Wing SL, Serre T. 2016. Computer vision cracks the

600 leaf code. Proceedings of the National Academy of Sciences: 201524473.

601 Wolpert DH, Macready WG. 1997. No free lunch theorems for optimization. IEEE

602 Transactions on Evolutionary Computation 1: 67-82.

603 Yu X, Liu B, Pei Y, Xu T. 2014. Evaluation of facial attractiveness for patients with

604 malocclusion: A machine-learning technique employing Procrustes. The Angle

605 Orthodontist 84: 410-416.

606 Zelditch ML, Swiderski DL, Sheets HD, Fink WL. 2004. Geometric morphometrics for 607 biologists. Oxford: Elsevier Academic Press.

608 Zhang L, Ampatzidis Y, Whiting MD. 2015. Sweet cherry floral organ size varies with 609 genotype and temperature. Scientia Horticulturae 182: 156-164. 